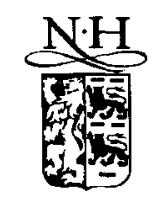

ELSEVIER

\title{
Compatibility of symplectic structures adapted to noncommutatively integrable systems
}

\author{
Francesco Fassò $^{\mathrm{a}, *}$, Tudor Ratiu ${ }^{\mathrm{b}, 1}$ \\ a Dipartimento di Matematica Pura e Applicata, Università di Padova, \\ Via G. Belzoni 7, 35131 Padova, Italy \\ ${ }^{\mathrm{b}}$ Mathematics Department, University of California, Santa Cruz, CA 95064, USA
}

Received 12 September 1997

\begin{abstract}
It is known that any integrable, possibly degenerate, Hamiltonian system is Hamiltonian relative to many different symplectic structures; under certain hypotheses, the 'semi-local' structure of these symplectic forms, written in local coordinates of action-angle type, is also known. The purpose of this paper is to characterize from the point of view of symplectic geometry the family of all these structures. The approach is based on the geometry of noncommutatively integrable systems and extends a recent treatment of the nondegenerate case by Bogoyavlenskij. Degenerate systems are comparatively richer in symplectic structures than nondegenerate ones and this has the counterpart that the bi-Hamiltonian property alone does not imply integrability. However, integrability is still guaranteed if a system is Hamiltonian with respect to three suitable symplectic structures. Moreover, some of the properties of recursion operators are retained. (O 1998 Elsevier Science B.V.
\end{abstract}

Subj. Class.: Dynamical systems

1991 MSC: 58F05

Keywords: Bi-Hamiltonian structures; Noncommutative integrability; Degenerate systems; Recursion operators

\footnotetext{
${ }^{*}$ Corresponding author. E-mail: fasso@math.unipd.it. Partially supported by the grant $E C$ contract ERBCHRXCT940460 for the project Stability and Universality in Classical Mechanics and, while visiting the Mathematics Department, University of California, Santa Cruz, CA 95064, by DOE contract DEFG0395ER25245-A000.

${ }^{1}$ E-mail: ratiu@math.ucsc.edu. Partially supported by NSF grant DMS-9503273 and DOE contract DEFG03-95ER25245-A000.
} 


\section{Introduction}

\section{1}

If a vector field $X$ on a manifold $M$ of dimension $2 d$ is Hamiltonian with respect to two different symplectic structures $\sigma_{0}$ and $\sigma_{1}$, then its flow preserves the eigenvalues of the recursion operator $R=\sigma_{0}^{\sharp} \sigma_{1}^{b}$; here $\sigma^{b}: T M \rightarrow T^{*} M$ is the isomorphism induced by the symplectic form $\sigma$ and $\sigma^{\sharp}$ is its inverse. Hence, if $R$ has $d$ eigenvalues which are functionally independent and pairwise in involution, then it is completely integrable. (Since $R$ is the product of two antisymmetric matrices, all its eigenvalues are at least double [1], and so at most $d$ are independent.)

Magri [2] showed that a sufficient condition for the eigenvalues of the recursion operator to be in involution is that the two symplectic structures are compatible, in the sense that the sum of the corresponding Poisson tensors is still a Poisson tensor. However, not all completely integrable systems are bi-Hamiltonian in Magri's sense, not even in a neighborhood of an invariant torus [3-5], or, as we shall say, semi-locally. (In this paper, 'semi-local' means always 'defined in a neighborhood of an entire invariant torus'.)

Recently, Bogoyavlenskij [6] has given another, broader, sufficient condition for the eigenvalues of the recursion operator to be in involution. This condition, which he calls strong dynamical compatibility, requires the existence of a vector field $X$ which is Hamiltonian with respect to both symplectic structures $\sigma_{0}$ and $\sigma_{1}$, is completely integrable with respect to one of them, and is anisochronous. Specifically, Bogoyavlenskij assumes that the orbits of $X$ lie on Lagrangian tori and that, in any local system of $\sigma_{0}$-action-angle coordinates $(a, \alpha)$, the $\sigma_{0}$-Hamiltonian $H(a)$ of $X$ satisfies at every point $a$ the anisochronicity condition $^{2}$

$$
\operatorname{det}\left(\frac{\partial^{2} H}{\partial a_{i} \partial a_{j}}\right)(a) \neq 0
$$

Condition (1.1) plays a central role in our context and is easily clarified. This condition implies that the dense subsets of the invariant $d$-dimensional tori of $X$ are closures of trajectories, so that every 'semi-local' integral of motion is constant on them. Together with the Lagrangian character of the tori, this implies that all semi-local integrals of motion of $X$ are in involution. On the other hand, the eigenvalues of the recursion operator are semi-local quantities (see also Section 3.1), and so one concludes that they are in involution.

Compared to Magri's, Bogoyavlenskij's notion of compatibility has the advantage that all completely integrable anisochronous systems are semi-locally bi-Hamiltonian in this sense. Furthermore, Bogoyavlenskij [6] could classify all semi-local symplectic structures which are compatible (in his own sense) with a given one, as well as all semi-local symplectic structures in which a given completely integrable anisochronous system is Hamiltonian. On

\footnotetext{
${ }^{2}$ Bogoyavlenskij uses the expression 'nondegenerate' instead of 'anisochronous', but we will use here the former with another meaning.
} 
the other hand, it can be difficult to practically verify the 'strong dynamical compatibility' of two given symplectic structures.

\section{2}

In this paper we study the case of degenerate (or superintegrable) systems, which have more than $d$ independent integrals of motion, say $2 d-n>d$ with some $n<d$, and correspondingly have motions which take place on isotropic tori of dimension $n$, rather than on Lagrangian tori of dimension $d$. This case includes classical systems like $d \geq 1$ harmonic oscillators with equal frequencies ( $n=1)$, the Kepler system $(d=3, n=1)$, and the free rigid body with a fixed point $(d=3, n=2)$. In [6] there are a few examples and partial results about the degenerate case (Theorem 8 and Sections 5 and 6); in particular, it is observed there that a degenerate system is Hamiltonian with respect to many more semilocal symplectic structures than a nondegenerate one. The expression of all these semi-local symplectic forms was given in [7], within a different context, and was also announced in $[18,19]$ as part of a proof of a more general result which we will mention later. Since this expression constitutes a prerequisite for the present work, we will review it in Section 2.2.

Our approach is based on the notion of noncommutative integrability, which appears to be the appropriate integrability notion for degenerate systems and is briefly reviewed in Section 2.1. In short, a Hamiltonian vector field is said to be noncommutatively integrable if it is tangent to the fibers of an isotropic fibration which, in addition, is symplectically complete, i.e., possesses a polar foliation. We shall moreover say that a system of this kind has $n$ frequencies if it possesses exactly $2 d-n$ semi-local integrals of motion near any invariant torus.

This point of view gives a thorough geometric insight, which explains the richness of symplectic structures of degenerate systems. As is expected, the reason is in the well-known fact that, in the degenerate case, the invariant isotropic tori can be grouped together so as to form infinitely many different Lagrangian foliations ([8, Section 51.D]; see also [9] for a discussion within the setting of noncommutative integrability), and as it turns out, each of them corresponds to a bi-symplectic structure.

More precisely, our purposes in the paper are the following:

(i) To give a (geometric) notion of compatible symplectic structures which is general enough to include all the semi-local symplectic structures in which a given noncommutatively integrable system with any number of frequencies is Hamiltonian. In turn, this will provide a geometric characterization of these semi-local symplectic structures. Specifically, motivated by the definition of noncommutative integrability, we shall introduce two distinct definitions of compatible symplectic structures. They both require the existence of a fibration whose fibers are 'bi-isotropic', have the same affine structures, and possess a polar with respect to both structures; for the stronger definition we also require that the two polars coincide (for details, see Section 3.1). The weaker definition includes all semi-local symplectic structures of a given degenerate system. However, the counterpart to the existence of more symplectic structures is that the property of being bi-Hamiltonian, even in the stronger sense, does not imply any more 
the noncommutative integrability of a degenerate system. From this point of view, the bi-Hamiltonian property appears to be similar to the Hamilton-Jacobi equation, namely a way of describing Lagrangian foliations not directly generalizable to the isotropic case. Nevertheless, it is possible to find three suitable symplectic structures such that any corresponding "tri-Hamiltonian" system is integrable in the noncommutative sense. We shall exemplify the situation in the simplest case of two harmonic oscillators with equal frequencies.

(ii) In the nondegenerate case, the above definition reduces to the existence of a fibration with compact connected fibers whose fibers are Lagrangian and have the same affine structure with respect to both symplectic structures. As we shall discuss, this notion of bi-Hamiltonian systems is more general than Bogoyavlenskij's, although retaining its main features regarding the relations between bi-Hamiltonianity and complete integrability (Section 3) and regarding the construction of symmetry by means of the recursion operator (Appendix A).

In the nondegenerate case, the ideas underlying this definition are not new. The relevance of bi-Lagrangian fibrations for the bi-Hamiltonian setting appears explicitly, for instance, in [5,10,11], and is also implicit in Bogoyavlenskij's definition of strong dynamical compatibility (the role of the nondegenerate bi-Hamiltonian vector field is precisely that of generating such a fibration). What seems instead to be new is the consideration of the degenerate, isotropic case.

(iii) Thirdly, at a more elementary level, we address here the problem of whether the independence of the eigenvalues of the recursion operator is really necessary to assure that all bi-Hamiltonian systems are completely (or noncommutatively, depending on the case) integrable. As we shall show by an example in Appendix A, this is not the case.

\section{3}

The paper is organized as follows. Section 2 is devoted to a short review of noncommutative integrability and provides the basis for the whole treatment. Section 3 deals with the definition of compatible symplectic structures and the characterization of the semi-local symplectic structures compatible to a given one. In Section 4 we investigate the relations between bi-Hamiltonianity and noncommutative integrability. Section 5 is devoted to the example. In Appendix A we consider some questions related to recursion operators and symmetries. Appendix B contains some comments about the adopted definition of compatible symplectic structures.

\section{Noncommutatively integrable systems and their semi-local structure}

\subsection{Noncommutative integrability}

We begin by recalling a few basic facts about the geometry of noncommutatively integrable systems (for more details, see $[9,12]$ ). 
Let $(M, \sigma)$ be a symplectic manifold of dimension $2 d$ and $\pi: M \rightarrow B$ a (locally trivial) fibration with fibers of dimension $n \leq d$ and base a manifold $B$ of dimension $2 d-n$. The polar foliation of $\pi$, if it exists, is the foliation whose tangent bundle is the distribution of the symplectic orthogonals to the tangent spaces of the fibers of $\pi$. If $\pi$ has a polar foliation, which we denote by $\pi^{\perp}$ (even though it is in general not a fibration), then $\pi$ is said to be symplectically complete.

We recall that a submanifold of $M$ is called isotropic (coisotropic) if its tangent spaces are contained in (contain) their own $\sigma$-orthogonals. A Lagrangian submanifold is one which is both isotropic and coisotropic. So, every Lagrangian fibration coincides with its own polar.

Symplectically complete fibrations, under various names (dual pairs, bifoliations), are well-studied objects (see [12-15]). We review here some of their basic properties:

(P1) A fibration $\pi: M \rightarrow B$ is symplectically complete if and only if $B$ has a Poisson structure $\Lambda$ such that $\pi:(M, \sigma) \rightarrow(B, \Lambda)$ is a Poisson morphism [14, Chapter 3, Proposition 9.7].

(P2) If $\pi: M \rightarrow B$ is symplectically complete and its fibers are isotropic, then they have an affine structure. More precisely, there exists a partial connection $\nabla^{(\sigma)}: \mathcal{X}_{\pi^{\perp}} \times \mathcal{X}_{\pi} \rightarrow \mathcal{X}_{\pi}$ on $M$, where $\mathcal{X}_{\pi}$ and $\mathcal{X}_{\pi \perp}$ are the spaces of all sections of $\pi$ and of its polar $\pi^{\perp}$, respectively. This partial connection is defined by

$$
\nabla_{X}^{(\sigma)} Y=\sigma^{\sharp} L_{X} \sigma^{b}(Y) \quad \forall X \in \mathcal{X}_{\pi^{\perp}}, \quad Y \in \mathcal{X}_{\pi},
$$

and its restriction $\nabla^{(\sigma)}: \mathcal{X}_{\pi} \times \mathcal{X}_{\pi} \rightarrow \mathcal{X}_{\pi}$ to the fibers of $\pi$ defines a flat torsion-free connection, whose existence implies that the compact connected components of the fibers of $\pi$ are diffeomorphic to $\mathbb{T}^{n}[16$, Section 4.6].

(P3) Under the same assumptions of ( $\mathrm{P} 2)$, every compact connected component of a fiber of $\pi$ has a neighborhood $N$ equipped with generalized action-angle coordinates, i.e., a diffeomorphism

$$
(b, \alpha): N \rightarrow U \times \mathbb{T}^{n},
$$

where $U \subset \mathbb{R}^{2 d-n}$ is open, such that $b$ is constant on the fibers of $\pi$, and writing $b=$ $(p, q, a): N \rightarrow \mathbb{R}^{d-n} \times \mathbb{R}^{d-n} \times \mathbb{R}^{n}$, one has

$$
\left.\sigma\right|_{N}=\sum_{i=1}^{d-n} \mathrm{~d} p_{i} \wedge \mathrm{d} q_{i}+\sum_{i=1}^{n} \mathrm{~d} a_{i} \wedge \mathrm{d} \alpha_{i}
$$

$(\operatorname{see}[12,15,17])$.

With the notation of (P3), the coordinates $a$ and $\alpha$ will be called, respectively, actions and angles; the $\alpha$ are (angular) coordinates on the fibers, and $b=(p, q, a)$ are local coordinates on the base $B$ of the fibration. Specifically, the actions $a$ are local Casimirs of $(B, \Lambda)$, while $(p, q)$ are local canonical coordinates on its symplectic leaves. Note also that the coisotropic 
leaves of the polar foliation are locally described by the equations $a=$ const. Hence, we have:

(P4) Any leaf of $\pi^{\perp}$ is projected by $\pi$ onto a symplectic leaf of $B$.

Finally, we explicitly note that, by (2.2), the Hamiltonian vector field of the action $a_{i}$ is $\partial / \partial \alpha_{i}(i=1, \ldots, n)$. As is clear, $\partial / \partial \alpha_{i}$ is tangent to the fibers of $\pi$ and has $2 \pi$ periodic orbits. Moreover, $\nabla_{\partial / \partial \alpha_{i}}^{(\sigma)} \partial / \partial \alpha_{j}=0$ for all $i, j$. This is verified by observing that $\nabla^{(\sigma)}$ has the following expression in generalized action-angle coordinates: if ${ }^{3} X=$ $X_{p_{u}} \partial / \partial p_{u}+X_{q_{u}} \partial / \partial q_{u}+X_{\alpha_{i}} \partial / \partial \alpha_{i}$ and $Y=Y_{\alpha_{i}} \partial / \partial \alpha_{i}$, then

$$
\nabla_{X}^{(\sigma)} Y=\left(L_{X} Y_{\alpha_{i}}\right) \frac{\partial}{\partial \alpha_{j}}
$$

Definition 1. (D1.1) A Hamiltonian vector field $X_{I I}$ on a symplectic manifold $(M, \sigma)$ is called noncommutatively integrable if it is tangent to the fibers of an isotropic symplectically complete fibration $\pi$ with compact and connected fibers.

(D1.2) If, moreover, the fibers of $\pi$ have dimension $n$ and $X_{H}$ does not possess more than $\operatorname{dim} M-n$ first integrals with differentials everywhere linearly independent in an open invariant set, then we say that $X_{H}$ has $n$ frequencies.

The above definition of noncommutative integrability is somewhat stricter than usual (one could only require the existence of an invariant foliation, rather than a fibration, with not necessarily compact leaves), but this is the case of interest here.

Under the hypotheses of (D1.1), in any system of generalized action-angle coordinates ( $p, q, a, \alpha$ ) of the fibration $\pi$, the Hamiltonian $H$ of $X$ is necessarily a function of the actions $a$ alone, $H=H(a)$. Hence, $X_{H}=\omega_{i}(a) \partial / \partial \alpha_{i}$ with $\omega=\partial H / \partial a$ and all its motions are quasi-periodic on the tori $b=$ const., i.e., on the fibers of $\pi$. Furthermore, under the hypotheses of (D1.2), $\pi$ is the finest invariant fibration of any open nonempty invariant set of $M$. In the following, we shall refer to such a fibration as the fibration by the invariant tori of $X_{H}$. We shall say that $X$ is anisochronous if $a \mapsto \omega(a)$ is everywhere a local diffeomorphism (i.e., condition (1.1) is satisfied).

When $n=d$, noncommutative integrability reduces to the usual notion of complete integrability, i.e., to the existence of a Lagrangian fibration with compact connected fibers diffeomorphic to $\mathbb{T}^{d}$, and of the corresponding action-angle coordinates $(a, \alpha)$. The novelty of the degenerate case is related to the existence of the polar foliation and to the Poisson structure on the base manifold, which is stratified into its own symplectic leaves.

In order to understand this structure, it may be useful to refer to Fig. 1 (after [9]). There, each 'daisy' represents a leaf $a=$ const. of the polar foliation, which is in turn fibered by the invariant tori $(p, q, a)=$ const. (its petals); each of them is based at a point $(p, q)$ of

\footnotetext{
${ }^{3}$ We understand everywhere the summation over repeated indices. Furthermore, we make the convention that the indices $i, j$ take the values $1, \ldots, n$, the indices $u, v$ the values $1, \ldots, n-d$, and the indices $r, s$ the values $1, \ldots, 2 d-n$.
} 


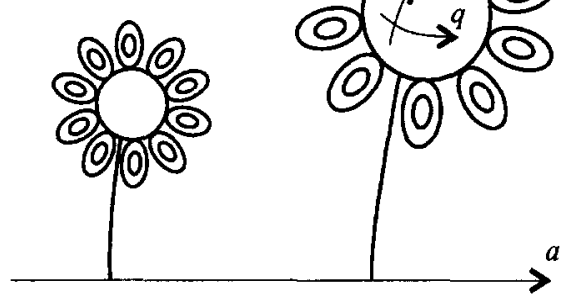

Fig. 1.

a symplectic leaf of the base manifold (the center of the daisy). Hence, all petals of the same daisy carry motions with equal frequencies $\omega(a)$. As an example, in the case of a system for which all motions are periodic $(n=1)$, the 'petals' are circles and the daisies are the connected components of the level sets of the Hamiltonian $H=H(a)$ (hence, the 'meadow' on which they grow is one-dimensional).

\subsection{The semi-local symplectic structures of noncommutatively integrable systems}

We consider now a Hamiltonian vector field $X$ on a symplectic manifold $\left(M, \sigma_{0}\right)$, which is noncommutatively integrable with $n$ frequencies. Introduce a system of local generalized action-angle coordinates $(b, \alpha)=(p, q, a, \alpha)$, so that $X=\omega_{i}(a) \partial / \partial \alpha_{i}$. In [7] (see also $[18,19]$ ) it has been shown that, in a neighborhood of any of its invariant tori, $X$ is Hamiltonian with respect to another symplectic structure $\sigma_{1}$ if and only if

$$
\sigma_{1}=E_{r i}(b) \mathrm{d} b_{r} \wedge \mathrm{d} \alpha_{i}+\frac{1}{2} F_{r s}(b) \mathrm{d} b_{r} \wedge \mathrm{d} b_{s}+\frac{1}{2} G_{i j} \mathrm{~d} \alpha_{i} \wedge \mathrm{d} \alpha_{j},
$$

where $E$ and $F=-F^{\mathrm{T}}$ are matrices which depend on $b, G=-G^{\mathrm{T}}$ is a constant matrix, $E$ and $G$ are such that

$$
\begin{aligned}
& \mathrm{d}\left(E_{r i} \omega_{i} \mathrm{~d} b_{r}\right)=0, \\
& G \omega=0,
\end{aligned}
$$

and $E$ and $F$ satisfy the obvious necessary conditions for $\sigma_{1}$ to be closed and nondegenerate.

The presence of the matrix $G$ in (2.4) is related to a delicate (but interesting) point. As is clear, the invariant tori of $X$ are $\sigma_{1}$-isotropic if and only if $G=0$ and, as it turns out, this is exactly the condition for $X$ to be $\sigma_{1}$-noncommutatively integrable [7]. Therefore, if $G \neq 0$, one has a Hamiltonian system with all motions quasi-periodic which is not completely (or noncommutatively) integrable. It is possible to give quite broad conditions which rule out this possibility, namely:

(C1) $\sigma_{1}$ is exact;

(C2) $n=1$ or $n=2$;

(C3) rank $\partial \omega / \partial a \geq n-1$ at some point of each connected component of the manifold. 
(See [7]; for $n=d$, conditions similar to (C3) were known to Gallavotti [26] and are given in Theorem 9 of [6]). Nevertheless, this possibility cannot be excluded, and systems of this kind do exist. A general framework for this situation, which seems to indicate that complete (or noncommutative) integrability might not be the ultimate integrability notion for Hamiltonian systems, is discussed in $[18,19]$. In the sequel, we restrict ourselves to the case $G=0$ in order to remain within the framework of noncommutative integrability.

If $G=0$, then it is not difficult to see (exploiting its closedness) that $\sigma_{1}$ has the form

$$
\sigma_{1}=\mathrm{d}\left(e_{i} \circ b\right) \wedge \mathrm{d} \alpha_{i}+\mathrm{d}\left(f_{r} \circ b\right) \wedge \mathrm{d} b_{r},
$$

where $e_{1}, \ldots, e_{n}$ and $f_{1}, \ldots, f_{2 d-n}$ are arbitrary functions subject only to the closedness condition

$$
\mathrm{d}\left(\omega_{i} \mathrm{~d}\left(e_{i} \circ b\right)\right)=0
$$

and to the nondegeneracy conditions

$$
\operatorname{rank} E=n, \quad \operatorname{rank}(F, E)=2 d-n,
$$

where the matrices $E$ and $F$, respectively, of dimensions $(2 d-n) \times n$ and $(2 d-n) \times(2 d-n)$, have entries

$$
E_{r i}=\frac{\partial e_{i}}{\partial b_{r}}, \quad F_{r s}=\frac{\partial f_{s}}{\partial b_{r}}-\frac{\partial f_{r}}{\partial b_{s}} ;
$$

note that the first equation in Eq. (2.8) implies that $e$ is a submersion.

Formula (2.6) implies that the $\sigma_{1}$-polar of the fibration $b=$ const. is described by the equations $e \circ b=$ const. (Indeed, a vector field $Z=Z^{b_{r}} \partial / \partial b_{r}+Z^{\alpha_{i}} \partial / \partial \alpha_{i}$ is tangent to the leaves of the polar foliation if and only if $0=\sigma_{1}\left(Z, \partial / \partial \alpha_{i}\right)=\partial e_{i} / \partial b_{r} Z^{b_{r}}=L_{Z} e_{i}$ for all $i$.) As a consequence, the $\sigma_{0}$ - and $\sigma_{1}$-polars of $\pi$ coincide if and only if the map e depends only on the actions $a$, in which case

$$
\sigma_{1}=\mathrm{d}\left(e_{i} \circ a\right) \wedge \mathrm{d} \alpha_{i}+\mathrm{d}\left(f_{r} \circ b\right) \wedge \mathrm{d} b_{r} .
$$

In particular, this case is met when $X$ is anisochronous, and under such a hypothesis, formula (2.10) further specializes. In fact, if $a \mapsto \omega(a)$ is a local diffeomorphism, then the closedness condition (2.7) can be written

$$
0=\mathrm{d}\left(e_{i}(b) \mathrm{d} \omega_{i}(a)\right)=\mathrm{d}\left(e_{i}(b) \frac{\partial \omega_{i}}{\partial a_{j}} \mathrm{~d} a_{j}\right)
$$

so that (locally) $e_{i} \partial \omega_{i} / \partial a_{j}=\partial g / \partial a_{j}$ for some function $g=g(a)$, which implies $e=e(a)$, as claimed. However, since one can regard $e$ and $g$ as functions of $\omega$, condition $\mathrm{d}\left(\mathrm{e}_{i} \mathrm{~d} \omega_{i}\right)=0$ implies

$$
e_{i}=\frac{\partial g}{\partial \omega_{i}}
$$

This is always the case if $\sigma_{0}$ and $\sigma_{1}$ are 'strongly dynamically compatible', as noted in [6]. 
We add a final comment. Any noncommutatively integrable system is obviously semilocally completely integrable, since the functions $I=(p, a)$ (for instance) are first integrals in involution. In many cases, the local coordinates $q_{1}, \ldots, q_{d-n}$ on the symplectic leaves can be taken to be angles, so that the generalized action-angle coordinates $(p, q, a, \alpha)$ become 'action-angle coordinates' $(I, \varphi)$, with $\varphi=(q, \alpha)$, adapted to the Lagrangian fibration $I=$ const. However, formulas (2.6) and (2.10) may contain also terms in $\mathrm{d} q_{i} \wedge \mathrm{d} q_{j}$, in which case the tori $I=$ const. are not $\sigma_{1}$-Lagrangian. Nevertheless, in the noncommutative approach, only the $\sigma_{1}$-isotropy of the smaller tori $(p, q, a)=$ const. matters, and this is a less stringent condition. The greater freedom relative to the terms in $\mathrm{d} p$ and $\mathrm{d} q$ in formulas (2.6) and (2.10) is connected with the possibility of changing canonical coordinates on the symplectic leaves of the base manifold; we shall exemplify this situation in Section 5 .

\section{Remarks.}

(i) To our knowledge, the semi-local characterization of Magri compatible symplectic forms is not known.

(ii) A particular symplectic structure of the form (2.10) for the Kepler system has been given in [20].

\section{Compatible symplectic structures}

\subsection{Definitions and semi-local characterization}

In this section we introduce two notions of compatible symplectic structures under which all symplectic structures (2.6) and (2.10), respectively, are compatible. These two notions coincide in the nondegenerate case $n=d$.

Definition 2. (D2.1) Let $M$ be a manifold of dimension $2 d$ and let $\sigma_{0}$ and $\sigma_{1}$ be two symplectic structures on it. Assume that there exists a fibration $\pi$ of $M$ with compact connected fibers of dimension $n \leq d$, which is:

- bi-isotropic (i.e., its fibers are isotropic with respect to $\sigma_{0}$ and $\sigma_{1}$ );

- bi-symplectically complete (i.e., $\pi$ has a polar with respect to $\sigma_{0}$ and $\sigma_{1}$ );

- bi-affine (i.e., the restrictions of $\nabla^{\left(\sigma_{0}\right)}$ and $\nabla^{\left(\sigma_{1}\right)}$ to the fibers of $\pi$ coincide).

Then, we say that $\left(\sigma_{0}, \sigma_{1}, \pi\right)$ form a bi-symplectic structure of order $n$ on $M$, or that $\sigma_{0}$ and $\sigma_{1}$ are $\pi$-compatible.

(D2.2) If, moreover, the $\sigma_{0}$ - and $\sigma_{1}$-polars of $\pi$ coincide, then we say that $\sigma_{0}$ and $\sigma_{1}$ are $\left(\pi, \pi^{\perp}\right)$-compatible.

(D2.3) We say that two symplectic structures $\sigma_{0}$ and $\sigma_{1}$ are independent if the recursion operator $R=\sigma_{0}^{\sharp} \sigma_{1}^{\mathrm{b}}$ has $d$ eigenvalues which are everywhere distinct and have everywhere linearly independent differentials. 
Some equivalent restatements of the condition of bi-affinity are given in Appendix B, where we also show in examples the independence of the three conditions entering (D2.1). We also note that the equality of the $\sigma_{0}$-and $\sigma_{1}$-polars of $\pi$ (as in (D2.2)) amounts to requiring the equality of the two partial connections $\nabla^{\left(\sigma_{0}\right)}$ and $\nabla^{\left(\sigma_{1}\right)}$ (not just of their restrictions to the fibers of $\pi$, as in (D2.1)).

The following proposition characterizes the semi-local structure of the symplectic forms which are $\pi$ - or $\left(\pi, \pi^{\perp}\right)$-compatible with a given one. This extends a result in [6] for the nondegenerate case.

\section{Proposition 1.}

(i) Let $\left(\sigma_{0}, \sigma_{1}, \pi\right)$ be a bi-symplectic structure of order $n$ on a manifold $M$ of dimension $2 d \geq 2 n$. Let $(b, \alpha)=(p, q, a, \alpha)$ be $\sigma_{0}$-generalized action-angle coordinates for $\pi$. Then, semi-locally, $\sigma_{1}$ has expressions (2.6)-(2.8). If $\sigma_{0}$ and $\sigma_{1}$ are also $\left(\pi, \pi^{\perp}\right)$ compatible, then $\sigma_{1}$ is as in (2.10).

(ii) Conversely, consider any open set $C \subset \mathbb{R}^{2 d-n}$, any submersion $e: C \rightarrow \mathbb{R}^{n}$, and any map $f: C \rightarrow \mathbb{R}^{2 d-n}$ fulfilling conditions (2.8) at any point of $C$. Let $\sigma_{0}$ and $\sigma_{1}$ be as in (2.2) and in (2.6), respectively, and let $\pi$ be the projection $(b, \alpha) \mapsto b$. Then, $\left(\sigma_{0}, \sigma_{1}, \pi\right)$ is a bi-symplectic structure of order $n$ on $C \times \mathbb{T}^{n}$. If, moreover, $C=Z \times A \subset \mathbb{R}^{2 d-2 n} \times \mathbb{R}^{n}$ and e is a submersion from $A$ into $\mathbb{R}^{n}$, then $\sigma_{0}$ and $\sigma_{1}$ are $\left(\pi, \pi^{\perp}\right)$-compatible.

Proof.

(i) In full generality, we can write

$$
\sigma_{1}=E_{r i}(b, \alpha) \mathrm{d} b_{r} \wedge \mathrm{d} \alpha_{i}+\frac{1}{2} F_{r s}(b, \alpha) \mathrm{d} b_{r} \wedge \mathrm{d} b_{s}+\frac{1}{2} G_{i j}(b, \alpha) \mathrm{d} \alpha_{i} \wedge \mathrm{d} \alpha_{j},
$$

where $E, F=-F^{\mathrm{T}}$ and $G=-G^{\mathrm{T}}$ are matrices which depend on $b$ and $\alpha$ and are $2 \pi$-periodic in $\alpha$. The $\sigma_{1}$-isotropy of the tori $b=$ const. amounts to $G=0$. Therefore, $\sigma_{1}^{b}\left(\partial / \partial \alpha_{i}\right)=-E_{r i} \mathrm{~d} b_{r}$ and so

$$
\begin{aligned}
0 & =\nabla_{\partial / \partial \alpha_{i}}^{\left(\sigma_{0}\right)} \partial / \partial \alpha_{j}=\nabla_{\partial / \partial \alpha_{i}}^{\left(\sigma_{1}\right)} \partial / \partial \alpha_{j} \\
& =\sigma_{1}^{\sharp}\left(L_{\partial / \partial \alpha_{i}}\left(-E_{r j} \mathrm{~d} b_{r}\right)\right)=-\sigma_{1}^{\sharp}\left(\frac{\partial E_{r j}}{\partial \alpha_{i}} \mathrm{~d} b_{r}\right)
\end{aligned}
$$

for any $i, j=1, \ldots, n$. Since $\sigma_{1}^{\sharp}$ is an isomorphism, this implies $\left(\partial E_{r j} / \partial \alpha_{i}\right) \mathrm{d} b_{r}=0$ for all $i, j$, i.e., $E=E(b)$. We now show that $F$ does not depend on the angles $\alpha_{i}$ either. The closedness of $\sigma_{1}$ implies

$$
0=\mathrm{d} \sigma_{1}\left(\frac{\partial}{\partial \alpha_{i}}, \frac{\partial}{\partial b_{r}}, \frac{\partial}{\partial b_{s}}\right)=\frac{\partial E_{r i}}{\partial b_{s}}-\frac{\partial E_{s i}}{\partial b_{r}}+\frac{\partial F_{r s}}{\partial \alpha_{i}} \quad \forall i, r, s,
$$

so that $\partial F_{r s} / \partial \alpha_{i}$ does not depend on $\alpha$; hence, since $F_{r s}$ is $2 \pi$-periodic in $\alpha$, one concludes that it does not depend on $\alpha$.

So far, we have proven that $\sigma_{1}$ has the form (2.4), with $G=0$. Hence, the closedness of $\sigma_{1}$ implies that the 1-forms $E_{r i} \mathrm{~d} b_{r}$ are closed, $i=1, \ldots, n$. Thus, in some open subset 
of the domain of the coordinates $b$ there exist $n$ functions $e_{i}$ such that $E_{r i} \mathrm{~d} b_{r}=\mathrm{d} e_{i}$. Similarly, $F_{r s} \mathrm{~d} b_{r} \wedge \mathrm{d} b_{s}$ is closed, and so $1 / 2 F_{r s} \mathrm{~d} b_{r}=\mathrm{d} f_{s}$ for some functions $f_{s}$. This proves that $\sigma_{1}$ has the form (2.6). The nondegeneracy of $\sigma_{1}$ implies that $\mathrm{d} e_{1}, \ldots$, $\mathrm{d} e_{n}$ are linearly independent at every point, i.e., that $e$ is a submersion.

Finally, if $\sigma_{0}$ and $\sigma_{1}$ have the same polar, then $0=\sigma_{1}\left(\partial / \partial z, \partial / \partial \alpha_{i}\right)=\partial e i / \partial z$ for all $z=p, q$ and all $i=1, \ldots, n$ so that $e=e(a)$.

(ii) In the coordinates $(b, \alpha), \sigma_{1}^{b}$ has the matrix

$$
\sigma_{1}^{b}=\left(\begin{array}{cc}
-F & -E \\
E^{\mathbf{T}} & 0
\end{array}\right)
$$

Since it has rank $2 d-n$ on account of (2.8), we conclude that $\sigma_{1}=\mathrm{d} e_{i} \wedge \mathrm{d} \alpha_{i}+\mathrm{d} f_{r} \wedge \mathrm{d} b_{r}$ is nondegenerate. It is obvious that the fibers of the projection $\pi: C \times \mathbb{T}^{n} \rightarrow C$ are isotropic with respect to both $\sigma_{0}$ and $\sigma_{1}$, and, as we have already mentioned, that its $\sigma_{0^{-}}$ and $\sigma_{1}$-polars are given by the equations $a=$ const. and $e(b)=$ const., respectively. The fact that the restrictions of $\nabla^{\left(\sigma_{0}\right)}$ and $\nabla^{\left(\sigma_{1}\right)}$ to the surfaces $b=$ const. (i.e., the fibers of $\pi$ ) agree, follows from

$$
\begin{aligned}
\nabla_{\partial / \partial \alpha_{i}}^{\left(\sigma_{1}\right)} \frac{\partial}{\partial \alpha_{j}} & =\sigma_{1}^{\sharp}\left(L_{\partial / \partial \alpha_{i}} \sigma_{1}^{b}\left(\frac{\partial}{\partial \alpha_{j}}\right)\right)=\sigma_{1}^{\sharp}\left(L_{\partial / \partial \alpha_{i}} \mathrm{~d} e_{j}\right) \\
& =\sigma_{1}^{\sharp}(0)=0, \quad i, j=1, \ldots, n
\end{aligned}
$$

(we used $e=e(b)$ ). The last statement is clear, since $e(a)=$ const. amounts to $a=$ const.

\section{Remarks.}

(i) Under the hypotheses of statement (i) of Proposition 1 , the functions $e_{1}, \ldots, e_{n}$ are $\sigma_{1}$-actions for the fibration $\pi$; one has indeed $\partial / \partial \alpha_{i}=X_{a_{i}}^{\sigma_{0}}=X_{e_{i}}^{\sigma_{1}}$. The corresponding angles are given by $\eta(b, \alpha)=\alpha-E^{-1} f(b)$.

(ii) Statement (ii) of Proposition 1 shows that, on a symplectic manifold, there are plenty of symplectic structures which are compatible (for any order) with a given one, at least semi-locally. As is obvious, globally it is another matter. (For some results on this point, in Magri's case, see [5].)

(iii) As an immediate consequence of Proposition 1 , one sees that, if $\left(\sigma_{0}, \sigma_{1}, \pi\right)$ is a bisymplectic structure on a manifold, then the eigenvalues of the recursion operator $R=\sigma_{0}^{\sharp} \sigma_{1}^{b}$ are defined in neighborhoods of the fibers of $\pi$. Indeed, it follows from (2.6) that the entries of the matrix representing $R$ in any system of generalized actionangle coordinates $(b, \alpha)$ of $\sigma_{0}$ depend only on $b$. (The exact expression of this matrix is given in formula (4.1).)

\subsection{Comparison with other compatibility notions}

We compare now the compatibility notion of Definition 2 with the one given by Bogoyavlenskij. First of all, we have the following: 


\section{Proposition 2.}

(i) Assume that a vector field $X$ on a manifold $M$ of dimension $2 d$ is Hamiltonian and noncommutatively integrable with respect to a symplectic structure $\sigma_{0}$ and that it has $n \leq d$ frequencies. Let $\pi$ be the fibration by the invariant tori of $X$. Assume also that $X$ is Hamiltonian relative to another symplectic structure $\sigma_{1}$ on $M$. Then

(i.1) If $X$ is $\sigma_{1}$-noncommutatively integrable (or else, if it satisfies any of the conditions (C1)-(C3) of Section 2.2), then $\sigma_{0}$ and $\sigma_{1}$ are $\pi$-compatible.

(i.2) If $n=d$, or if $X$ is anisochronous, then $\sigma_{0}$ and $\sigma_{1}$ are $\left(\pi, \pi^{\perp}\right)$-compatible.

(ii) Let $\left(\sigma_{0}, \sigma_{1}, \pi\right)$ be a bi-symplectic structure of order $n$ on a manifold $M$ of dimension $2 d \geq 2 n$. Then, for every point $m \in M$ there exists a neighborhood $C$ of $\pi(m)$ and a vector field $X$ on $\pi^{-1}(C)$ which is tangent to the fibers of $\pi$, is Hamiltonian and noncommutatively integrable with respect to both $\sigma_{0}$ and $\sigma_{1}$, and has $n$ frequencies.

Proof.

(i.1) If $X$ is $\sigma_{1}$-noncommutatively integrable, then $\sigma_{1}$ has the form (2.6) in every system of $\sigma_{0}$-generalized action-angle coordinates of $X$. Hence, by statement (ii) of Proposition $1, \sigma_{0}$ and $\sigma_{1}$ are $\pi$-compatible. Any of the conditions (C1)-(C3) is sufficient for $X$ to be $\sigma_{1}$-noncommutatively integrable.

(i.2) If $n=d$, the two polars coincide. The same happens when $a \mapsto \omega(a)$ is a local diffeomorphism, since then, as observed in Section 2.2, $\sigma_{1}$ has the form (2.10) with $e=e(a)$.

(ii) Consider any vector $\omega=\left(\omega_{1}, \ldots, \omega_{n}\right) \in \mathbb{R}^{n}$ which is nonresonant (i.e., $\omega \cdot v \neq 0$ for all $\left.\nu \in \mathbb{Z}^{n} \backslash\{0\}\right)$. Then, in any domain of generalized action-angle coordinates $(b, \alpha)$ of $\left(\pi, \sigma_{0}\right)$, the vector field $\sum_{i} \omega_{i} \partial / \partial \alpha_{i}$ has all the required properties.

Statement (i.2) shows that any two symplectic structures which are 'strongly dynamically compatible' in Bogoyavlenskij's sense are also $\pi$-compatible for some Lagrangian fibration $\pi$. So, in the nondegenerate case $n=d$, the novelty of our definition resides in that we relax the anisochronicity condition (1.1), replacing it with the assumption that the vector field has $d$ frequencies. This actually produces a more general definition:

Proposition 3. Let

$$
e_{1}\left(a_{1}, a_{2}\right)=a_{1}^{2} a_{2}, \quad e_{2}\left(a_{1}, a_{2}\right)=a_{1} a_{2},
$$

and consider the two symplectic forms $\sigma_{0}=\sum_{i=1}^{2} \mathrm{~d} a_{i} \wedge \mathrm{d} \alpha_{i}$ and $\sigma_{1}=\sum_{i=1}^{2} \mathrm{~d} e_{i} \wedge \mathrm{d} \alpha_{i}$ on $M=\mathbb{R}_{+}^{2} \times \mathbb{U}^{2}$, which are independent in an open nonempty subset $A \times \mathbb{J}^{2}$ of $M$. Then:

(i) $\sigma_{0}$ and $\sigma_{1}$ are not strongly dynamically compatible in the sense of [6].

(ii) Let $\pi(a, \alpha)=a$. Then $\left(\sigma_{0}, \sigma_{1}, \pi\right)$ is a bi-symplectic structure of order 2 on $M$.

Proof. Since $n=d$, the eigenvalues of the recursion operator coincide with those of the matrix $E$ (see Eq. (4.1)), which are $\left(2 a_{2}+1 \pm \sqrt{4 a_{2}^{2}+1}\right) a_{1} / 2$, proving the independence. As observed in Section 2.2, if $\sigma_{0}$ and $\sigma_{1}$ are strongly dynamically compatible, then $a=a(\omega)$ and the local diffeomorphism $e=e(a(\omega))$ entering (2.10) has the form $e=\partial g / \partial \omega$ for 
some function $g(\omega)$. If $n=2$, as one verifies using the identity $\partial / \partial a_{i}=\left(\partial \omega_{j} / \partial a_{i}\right)\left(\partial / \partial \omega_{j}\right)$, this implies

$$
\frac{\partial^{2} e_{1}}{\partial a_{1} \partial a_{2}}=\frac{\partial^{2} e_{2}}{\partial a_{1} \partial a_{1}} .
$$

This equality is not fulfilled for $e_{1}$ and $e_{2}$ given in (3.1), which proves statement (i). Statement (ii) follows from statement (ii) of Proposition 1 , since det $\partial e / \partial a=a_{1}^{2} a_{2}$.

It is not known to us whether our definition (as well as Bogoyavlenskij's) is more general than Magri's. If $\sigma_{0}$ and $\sigma_{1}$ are Magri compatible, then the eigenvalues of the recursion operator (if independent) define a bi-Lagrangian foliation. However, even when this foliation is a fibration and has compact and connected fibers, it is not clear whether, using the terminology of Definition 2, it is bi-affine; as noticed by various authors, this is equivalent to the existence of an anisochronous completely integrable bi-Hamiltonian vector field (see [5] and also Proposition B.1 in Appendix B). On the other hand, we mention that there exist strongly dynamically compatible structures which are not Magri compatible [6, Section 5].

\section{Bi-Hamiltonian systems and noncommutative integrability}

\section{1}

We consider now the central problem for the bi-Hamiltonian setting, namely, whether the property of being bi-Hamiltonian implies the (noncommutative) integrability of a system.

Proposition 4. Let $\sigma_{0}$ and $\sigma_{1}$ be independent and $\left(\pi, \pi^{\perp}\right)$-compatible. Then, any vector field $X$ which is $\sigma_{0}$ - and $\sigma_{1}$-Hamiltonian is tangent to the leaves of the foliation polar to $\pi$.

Proof. In the $\sigma_{0}$-generalized action-angle coordinates $(b, \alpha)$ of $\pi$, the recursion operator has the structure

$$
R=\left(\begin{array}{cccc}
F_{p q}^{\mathrm{T}} & -F_{q q} & -F_{q a} & -E_{q \alpha} \\
F_{p p} & F_{p q} & F_{p a} & E_{p \alpha} \\
E_{p \alpha}^{\mathrm{T}} & E_{q \alpha}^{\mathrm{T}} & E_{a \alpha}^{\mathrm{T}} & 0 \\
-F_{p a}^{\mathrm{T}} & -F_{q a}^{\mathrm{T}} & F_{a a} & E_{a \alpha}
\end{array}\right),
$$

where $E$ and $F$ are as in (2.9) and we have ordered their entries according to the order of the coordinates $b=(p, q, a)$, namely:

- $F_{p p}, F_{p q}$, and $F_{q q}$ are $(d-n) \times(d-n)$ matrices with entries $F_{u v}, F_{u, d-n+v}$ and $F_{d-n+u, d-n+v}$, respectively, $(u, v=1, \ldots, d-n)$;

- $E_{p \alpha}$ and $E_{q \alpha}$ are $(d-n) \times n$ matrices with entries $E_{u, 2 d-2 n+j}$ and $E_{d-n+u, 2 d-2 n+j}$, respectively, $(u=1, \ldots, d-n, j=1, \ldots, n)$;

- $E_{a \alpha}$ and $F_{a a}$ are $n \times n$ matrices with entries $E_{2 d-2 n+i, 2 d-2 n+j}$ and $F_{2 d-2 n+i, 2 d-2 n+j}$, respectively, $(i, j=1, \ldots, n)$. 
Since under the present hypothesis $e=e(a)$, then $E_{q \alpha}=E_{p \alpha}=0$ and so the eigenvalue problem for $R$ factorizes. Specifically, the eigenvalues of $R$ are those of $E=E_{a \alpha}$, each counted twice, and those of the matrix

$$
\left(\begin{array}{cc}
F_{p q}^{\mathrm{T}} & -F_{q q} \\
F_{p p} & F_{p q}
\end{array}\right),
$$

which also are all double because it is the product of two antisymmetric matrices. Since by hypothesis $R$ has $d$ distinct and independent eigenvalues, it follows that the matrix $E$ has $n$ distinct and independent eigenvalues. Therefore, observing that $E$ depends only on $a$, one concludes that its eigenvalues are locally invertible functions of the actions $a$. The proof is concluded by recalling that the level sets of the actions are the leaves of the foliation polar to $\pi$.

Proposition 4 shows that, if $\sigma_{0}$ and $\sigma_{1}$ are $\pi$-compatible and $\pi$ is Lagrangian (i.e., $\pi=$ $\pi^{\perp}$ ), then any bi-Hamiltonian vector field is completely integrable with respect to both $\sigma_{0}$ and $\sigma_{1}$.

The conclusion of Proposition 4 is instead much poorer if $\sigma_{0}$ and $\sigma_{1}$ are $\left(\pi, \pi^{\perp}\right)$ compatible, with $\pi$ isotropic but not Lagrangian. The conclusion is that any bi-Hamiltonian vector field is tangent to the leaves of $\pi^{\perp}$, rather than to those of $\pi$. We do not know whether this result can be improved. The point is that the only ingredient used in the proof is the very general fact that a bi-Hamiltonian vector field preserves the $d$ eigenvalues of the recursion operator, but there could be additional constraints (see Remark (i) below). However, one cannot expect, in general, that any bi-Hamiltonian vector field is tangent to the fibers of $\pi$, as is seen in the following example:

$$
\begin{aligned}
& \sigma_{0}=\mathrm{d} a \wedge \mathrm{d} \alpha+\mathrm{d} p \wedge \mathrm{d} q, \quad \sigma_{1}=\mathrm{d} a^{2} \wedge \mathrm{d} \alpha+\mathrm{d} p^{2} \wedge \mathrm{d} q, \\
& \pi(p, q, a, \alpha)=(p, q, a) .
\end{aligned}
$$

The two symplectic forms $\sigma_{0}$ and $\sigma_{1}$ are $\left(\pi, \pi^{\perp}\right)$-compatible of order 1 on $\mathbb{R}_{+}^{3} \times S^{1} \ni$ $(p, q, a, \alpha)$ and are independent (the eigenvalues of the recursion operator being $a$ and $p$ ). Nevertheless, the vector field $\partial / \partial q$ is $\sigma_{0}$ - and $\sigma_{1}$-Hamiltonian (with Hamilton functions $p$ and $p^{2}$, respectively) but not tangent to the fibers of $\pi$.

\section{Remarks.}

(i) One can ask whether the conservation of the eigenvalues of the recursion operator is the only constraint that the bi-Hamiltonian character imposes on a vector field. As we show in Appendix A.2 by a particular example, this does not seem to be the case: there exist compatible structures which are not independent and nevertheless have the property that any bi-Hamiltonian vector field is completely integrable. This situation is in our opinion very interesting and warrants further study.

(ii) In principle, if $\pi$ is not Lagrangian, Proposition 4 might not even exclude the existence of systems which are bi-Hamiltonian and nevertheless truly nonintegrable. In fact, if the eigenvalues of the recursion operator are not in involution, their conservation only implies that any bi-Hamiltonian vector field has as many independent integrals of 
motion as degrees of freedom, a property which, without the involutivity, does not rule out the possibility of chaotic motions (an example is the four vortices system [21-23]). However, we do not know if systems with this strange property do exist.

\subsection{Tri-Hamiltonian systems}

Even though the bi-Hamiltonian character relative to a bi-symplectic structure of order $n<d$ does not imply complete (or noncommutative) integrability, there is still a possibility to produce isotropic invariant foliations, namely, that the system be Hamiltonian with respect to three symplectic structures with suitable properties.

The simplest situation occurs when there are two pairs of independent bi-symplectic structures of order $d$, say $\left(\sigma_{0}, \sigma_{1}, \pi_{1}\right)$ and $\left(\sigma_{0}, \sigma_{2}, \pi_{2}\right)$, with different $\sigma_{0}$-Lagrangian fibrations $\pi_{1}$ and $\pi_{2}$. In this case, any 'tri-Hamiltonian' vector field has to be tangent to the intersection of the fibers of these two fibrations. So, if these submanifolds are isotropic and have a polar foliation, any 'tri-Hamiltonian' vector field is noncommutatively integrable.

As we now show, in typical situations, if $X$ is noncommutatively integrable and has $n$ frequencies, one can always find (at least semi-locally) three symplectic structures as above in such a way that $\pi_{1}$ and $\pi_{2}$ intersect exactly on the invariant tori of $X$. Specifically, let us refer to the situation in which $X$ can be described, at least locally, as a completely integrable system, i.e., the local coordinates $q_{1}, \ldots, q_{d-n}$ on the symplectic leaves can be taken to be angles, so that the surfaces $(p, a)=$ const. are Lagrangian tori. In order to construct, at least locally, a second Lagrangian fibration, we can for instance introduce new local 'action-angle' coordinates $\left(p^{\prime}, q^{\prime}\right)$ on the symplectic leaves by

$$
p_{u}^{\prime}=\left(1+\frac{1}{2} \cos q_{u}\right) p_{u}, \quad q_{u}^{\prime}=q_{u}+\frac{1}{2} \sin q_{u}
$$

$(u=1, \ldots, d-n)$, so that the coordinate circles $q_{u}$ and $q_{u}^{\prime}$ are different and

$$
\sigma_{0}=\mathrm{d} a_{i} \wedge \mathrm{d} \alpha_{i}+\mathrm{d} p_{u} \wedge \mathrm{d} q_{u}=\mathrm{d} a_{i} \wedge \mathrm{d} \alpha_{i}+\mathrm{d} p_{u}^{\prime} \wedge \mathrm{d} q_{u}^{\prime} .
$$

Therefore, as one immediately sees,

$$
\begin{aligned}
& \sigma_{1}=\mathrm{d} a_{i}^{2} \wedge \mathrm{d} \alpha_{i}+\mathrm{d} p_{u}^{2} \wedge \mathrm{d} q_{u}, \quad \pi_{1}(p, q, a, \alpha)=(p, a), \\
& \sigma_{2}=\mathrm{d} a_{i}^{2} \wedge \mathrm{d} \alpha_{i}+\mathrm{d} p_{u}^{\prime 2} \wedge \mathrm{d} q_{u}^{\prime}, \quad \pi_{2}\left(p^{\prime}, q^{\prime}, a, \alpha\right)=\left(p^{\prime}, a\right),
\end{aligned}
$$

have all the required properties. We shall exhibit a slightly different, but similar, situation in the next section.

\section{Example}

We consider here the classical system of two harmonic oscillators with equal frequencies. The Hamiltonian is given by

$$
H\left(x_{1}, x_{2}, y_{1}, y_{2}\right)=\frac{1}{2}\left(x_{1}^{2}+y_{1}^{2}+x_{2}^{2}+y_{2}^{2}\right)
$$


on $\mathbb{R}^{4} \approx \mathbb{C}^{2}$ with the 2-form $\sum_{i} \mathrm{~d} x_{i} \wedge \mathrm{d} y_{i}$. The system has all orbits periodic with period $2 \pi$, except for the equilibrium, which will be tacitly excluded from the considerations below. The periodic orbits are the fibers of a fibration $\pi$ which can be described, for instance, by the three integrals of motion

$$
F_{1}=y_{1} y_{2}+x_{1} x_{2}, \quad F_{2}=x_{1} y_{2}-x_{2} y_{1}, \quad F_{3}=\frac{1}{2}\left(x_{1}^{2}+y_{1}^{2}-x_{2}^{2}-y_{2}^{2}\right)
$$

(see e.g. [12]). $F_{1}, F_{2}, F_{3}$ are the components of the momentum map of the $S U(2)$-action on $\mathbb{C}^{2}$.

Specifically, $\pi=\left(F_{1}, F_{2}, F_{3}\right)$ is a submersion of $M=\mathbb{R}^{4} \backslash\{0\} \approx S^{3} \times \mathbb{R}_{+}$onto $B=$ $\mathbb{R}^{3} \backslash\{0\} \approx S^{2} \times \mathbb{R}_{+}$, with fiber $S^{1}$. The leaves of its polar foliation $\pi^{\perp}$ are the level sets of the Hamiltonian $H$, which are diffeomorphic to $S^{3}$. Hence, by property (P4) in Section 2.1, the symplectic leaves of $B$ are the two-dimensional spheres $\left\{F \in \mathbb{R}^{3}:\|F\|=H\right\}$. As is well known, the restriction of $\pi$ to the level sets of $H$ is the Hopf fibration $S^{3} \rightarrow S^{2}$.

We construct now a set of (local) generalized action-angle coordinates of the fibration $\pi: M \rightarrow B$. Note that the standard 'action-angle' coordinates $\left(I_{1}, I_{2}, \varphi_{1}, \varphi_{2}\right)$, with $x_{i}=$ $\sqrt{2 I_{i}} \cos \varphi_{i}, y_{i}=\sqrt{2 I_{i}} \sin \varphi_{i}$, are not generalized action-angle coordinates of $\pi$, since $H=I_{1}+I_{2}$. However, a system of generalized action-angle coordinates is constructed out of them by taking

$$
a=I_{1}+I_{2}, \quad \alpha=\varphi_{1}, \quad p=-I_{2}, \quad q=\varphi_{1}-\varphi_{2},
$$

so that $H=a$. In these coordinates, the first integrals (5.2) become

$$
F_{1}=2 \sqrt{-p(a+p)} \cos q, \quad F_{2}=2 \sqrt{-p(a+p)} \sin q, \quad F_{3}=a+2 p,
$$

so that $(2 \sqrt{-p(a+p)}, q, a+2 p)$ are cylindrical coordinates on the base $B=\mathbb{R}^{3} \backslash\{0\}$. This shows that the coordinates $(p, q)$ on the symplectic leaves $\|F\|=a$ are cylindric-like coordinates relative to the base $\left(F_{1}, F_{2}, F_{3}\right)$, with singularities on the axis $F_{3}$. The presence of some singularities of the generalized action-angle coordinates on any symplectic leaf is unavoidable, since they are compact (and moreover, the Hopf fibration is nontrivial). However, the location of these singularities can be chosen at will. This should actually be obvious, since, on account of the isotropy of the system, the periodic orbits in which only one of the two oscillators moves are not different from all other orbits.

To show this in a formal way, we construct a new set of generalized action-angle coordinates. To this end, we begin by making a rotation by an angle $\psi$ in the plane $\left(y_{1}, y_{2}\right)$, obtaining the new coordinates

$$
y_{1}^{\prime}=y_{1} \cos \psi-y_{2} \sin \psi, \quad y_{2}^{\prime}=y_{1} \sin \psi+y_{2} \cos \psi,
$$

and we canonically extend this change of coordinates to the momenta

$$
x_{1}^{\prime}=x_{1} \cos \psi-x_{2} \sin \psi, \quad x_{2}^{\prime}=x_{1} \sin \psi+x_{2} \cos \psi .
$$

In the coordinates $\left(x^{\prime}, y^{\prime}\right)$ on $\mathbb{R}^{4}$, the Hamiltonian retains its form $\left(x_{1}^{\prime 2}+y_{1}^{\prime 2}+x_{2}^{\prime 2}+y_{2}^{\prime 2}\right) / 2$, and so the fibration by the periodic orbits can also be described by the three first integrals

$$
F_{1}^{\prime}=y_{1}^{\prime} y_{2}^{\prime}+x_{1}^{\prime} x_{2}^{\prime}, \quad F_{2}^{\prime}=x_{1}^{\prime} y_{2}^{\prime}-x_{2}^{\prime} y_{1}^{\prime}, \quad F_{3}^{\prime}=\frac{1}{2}\left(x_{1}^{\prime 2}+y_{1}^{\prime 2}-x_{2}^{\prime 2}-y_{2}^{\prime 2}\right) \text {. }
$$




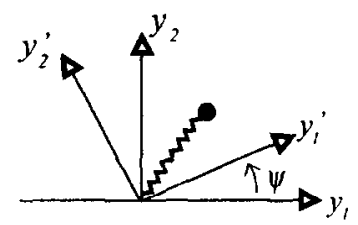

(a)
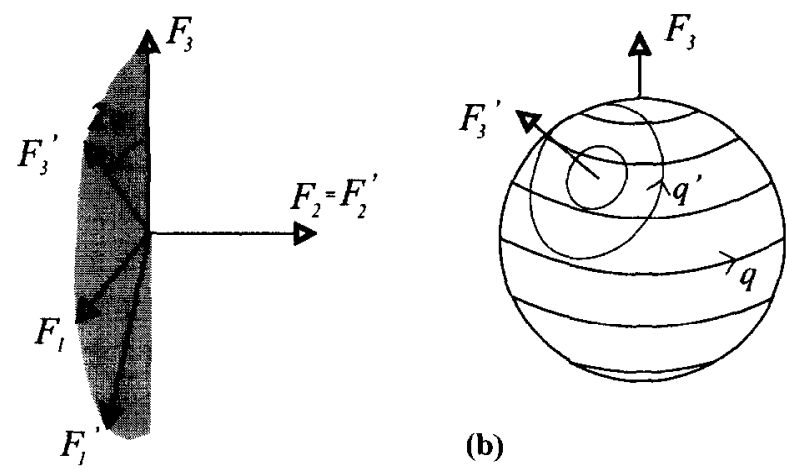

(b)

Fig. 2.

Hence, $\left(F_{1}^{\prime}, F_{2}^{\prime}, F_{3}^{\prime}\right)$ can be used as new coordinates on the base $B$ of the fibration. Specifically, as some algebra shows, the coordinates $F^{\prime}$ are related to the coordinates $F$ by a rotation by an angle $2 \psi$ around the axis $F_{2}$ (see Fig. 2(a)).

Now, proceeding exactly as above, we introduce action-angle coordinates $\left(I_{1}^{\prime}, I_{2}^{\prime}, \varphi_{1}^{\prime}, \varphi_{2}^{\prime}\right)$ with $x_{i}^{\prime}=\sqrt{2 I_{i}^{\prime}} \cos \varphi_{i}^{\prime}, y_{i}^{\prime}=\sqrt{2 I_{i}^{\prime}} \sin \varphi_{i}^{\prime}$ and we construct out of them the generalized action-angle coordinates

$$
a^{\prime}=I_{1}^{\prime}+I_{2}^{\prime}, \quad \alpha^{\prime}=\varphi_{1}^{\prime}, \quad p^{\prime}=-I_{2}^{\prime}, \quad q^{\prime}=\varphi_{1}^{\prime}-\varphi_{2}^{\prime} .
$$

Note that $a^{\prime}=a$, while $\left(p^{\prime}, q^{\prime}\right)$ are new coordinates on the symplectic leaves of the base manifold. Precisely, $\left(p^{\prime}, q^{\prime}\right)$ are cylindric-like coordinates relative to the rotated coordinates $\left(F_{1}^{\prime}, F_{2}^{\prime}, F_{3}^{\prime}\right)$, with singularities on the axis $F_{3}^{\prime}$, not on the axis $F_{3}$.

On every symplectic leaf, the curves $p=$ const. and $p^{\prime}=$ const. are circles forming an angle $2 \psi$, as illustrated in Fig. 2(b). Correspondingly, the two Lagrangian fibrations $I=$ const. and $I^{\prime}=$ const. exactly intersect on the periodic orbits. By means of these two Lagrangian fibrations and of the symplectic structures $\sigma_{0}, \sigma_{1}$, and $\sigma_{2}$ as in (4.3) and (4.4), one obtains a tri-Hamiltonian structure for the system.

Finally, we mention that similar situations are met in the free rigid body with a fixed point and in the Kepler system; the details can be reconstructed from the treatments of these systems given in [9] and [24], respectively.

\section{Appendix A}

In this appendix we discuss some questions related to recursion operators.

\section{A.1}

One of the distinct features of the standard bi-Hamiltonian approach (see $[2,25])$ is that by repeatedly applying the recursion operator to a bi-Hamiltonian system one obtains new bi-Hamiltonian systems, whose Hamiltonians are first integrals in involution of the original 
system. Only part of this survives in the present approach, just as in [6], and only if we refer to the stronger notion of $\left(\pi, \pi^{\perp}\right)$-compatibility. Specifically, the recursion operator produces only symmetries, which could be not Hamiltonian and, in degenerate cases, might even not commute.

Proposition A.1. Assume that $\sigma_{0}$ and $\sigma_{1}$ are $\left(\pi, \pi^{\perp}\right)$-compatible of order $n$ and independent. Let $R=\sigma_{0}^{\sharp} \sigma_{1}^{b}$ and assume that $X$ is $\sigma_{0}$-and $\sigma_{1}$-Hamiltonian. Then:

(i) $R^{k} X$ is tangent to the leaves of $\pi^{\perp}$ for any $k \geq 1$.

(ii) If $n=d$, then $R^{k} X$ is tangent to the fibers of $\pi$, has linear flow, and $\left[R^{k} X, R^{l} X\right]=0$ $(k, l=1, \ldots, n)$.

Proof. If $X$ is bi-Hamiltonian, then, by statement (ii) of Proposition 4, it is tangent to the leaves of $\pi^{\perp}$. Hence, in order to prove statement (i), it suffices to prove that, under the stated hypotheses, the tangent spaces to the leaves of $\pi^{\perp}$ are invariant under $R$. This is proven by observing that, since $\sigma_{1}$ has expression (2.10) with $e=e(a)$, in expression (4.1) of the recursion operator one has $E_{q \alpha}=E_{p \alpha}=0$, so that vectors with zero $\alpha$-components are mapped into vectors with zero $\alpha$-components.

If $n=d$, so that $\pi$ coincides with its polar, then $X=\omega_{i}(a) \partial / \partial \alpha_{i}$ by Proposition 4 . Hence, using (4.1), one sees that $R^{k} X=\left(E_{a \alpha}^{k}\right)_{i j} \omega_{j} \partial / \partial \alpha_{i}$, which is constant on each torus $a=$ const. Commutativity follows from here.

\section{A.2}

As anticipated in Section 4.1, we show now by an example that, given two symplectic structures $\sigma_{0}$ and $\sigma_{1}$, it may happen that all bi-Hamiltonian systems are bi-completely integrable even though the eigenvalues of $R=\sigma_{0}^{\sharp} \sigma_{1}^{\mathrm{b}}$ are not independent.

Specifically, let us consider the two symplectic structures

$$
\sigma_{0}=\mathrm{d} a_{1} \wedge \mathrm{d} \alpha_{1}+\mathrm{d} a_{2} \wedge \mathrm{d} \alpha_{2}, \quad \sigma_{1}=\mathrm{d}\left(\frac{a_{2}^{2}}{2}\right) \wedge \mathrm{d} \alpha_{1}+\mathrm{d}\left(\frac{a_{1}^{2}}{2}\right) \wedge \mathrm{d} \alpha_{2}
$$

on $\mathbb{R}_{+}^{2} \times \mathbb{T}^{2} \ni(a, \alpha)$. The matrices $E$ and $F$ defined in (2.9) are in this case

$$
E=\left(\begin{array}{cc}
0 & a_{1} \\
a_{2} & 0
\end{array}\right), \quad F=0
$$

and the eigenvalues of the recursion operator are $\pm \sqrt{a_{1} a_{2}}$, which are not independent. However, as we now show, any vector field which is $\sigma_{0}$ - and $\sigma_{1}$-Hamiltonian is necessarily tangent to the tori $a=$ const. and hence is completely integrable.

To this end, let us first note that, in general, if $\sigma_{0}$ and $\sigma_{1}$ are as in (2.2) and (2.10) with $n=d$, and if $H$ and $K$ are the $\sigma_{0}$ - and $\sigma_{1}$-Hamiltonians of a vector field $X=$ $X^{a_{i}} \partial / \partial a_{i}+X^{\alpha_{i}} \partial / \partial \alpha_{i}$, respectively, then one has

$$
\frac{\partial K}{\partial \alpha}=E^{\mathrm{T}} \frac{\partial H}{\partial \alpha}, \quad \frac{\partial K}{\partial a}=E \frac{\partial H}{\partial a}+F \frac{\partial H}{\partial \alpha} .
$$


In the present case, these equations read

$$
\frac{\partial K}{\partial \alpha_{1}}=a_{2} \frac{\partial H}{\partial \alpha_{2}}, \quad \frac{\partial K}{\partial \alpha_{2}}=a_{1} \frac{\partial H}{\partial \alpha_{1}}, \quad \frac{\partial K}{\partial a_{1}}=a_{1} \frac{\partial H}{\partial a_{2}}, \quad \frac{\partial K}{\partial a_{2}}=a_{2} \frac{\partial H}{\partial a_{1}} .
$$

Hence, equating the mixed derivatives of $K$ with respect to $\alpha_{1}$ and $\alpha_{2}$ one obtains

$$
a_{1} \frac{\partial^{2} H}{\partial \alpha_{1}^{2}}=a_{2} \frac{\partial^{2} H}{\partial \alpha_{2}^{2}}
$$

Similarly, after equating the mixed derivatives of $K$ with respect to $a_{1}$ and $\alpha_{2}$ and with respect to $a_{2}$ and $\alpha_{1}$, respectively, one obtains

$$
a_{1} \frac{\partial^{2} H}{\partial \alpha_{2} \partial a_{2}}=\frac{\partial H}{\partial \alpha_{1}}+a_{1} \frac{\partial^{2} H}{\partial \alpha_{1} \partial a_{1}}, \quad a_{2} \frac{\partial^{2} H}{\partial \alpha_{1} \partial a_{1}}=\frac{\partial H}{\partial \alpha_{2}}+a_{2} \frac{\partial^{2} H}{\partial \alpha_{2} \partial a_{2}},
$$

so that

$$
a_{2} \frac{\partial H}{\partial \alpha_{1}}=a_{1} \frac{\partial H}{\partial \alpha_{2}}
$$

Using Eq. (A.1) once and then Eq. (A.2) twice, one obtains

$$
\frac{\partial^{2} H}{\partial \alpha_{1}^{2}}=\frac{a_{2}}{a_{1}} \frac{\partial^{2} H}{\partial \alpha_{2}^{2}}=\left(\frac{a_{2}}{a_{1}}\right)^{2} \frac{\partial^{2} H}{\partial \alpha_{2} \partial \alpha_{1}}=\left(\frac{a_{2}}{a_{1}}\right)^{3} \frac{\partial^{2} H}{\partial \alpha_{1}^{2}},
$$

so that $\partial^{2} H / \partial \alpha_{1}^{2}=0$. Hence, by (A.1), $\partial^{2} H / \partial \alpha_{2}^{2}=0$. Since $H$ must be a periodic function of the angles, this shows that $H$ is independent of $\alpha_{1}$ and $\alpha_{2}$, i.e., $H=H(a)$ is completely integrable, as claimed.

\section{A.3}

In [20], Marmo and Vilasi proved the following: if a Hamiltonian vector field $X$ has all its orbits periodic and if the period-energy relation is smooth and does not have any critical points, then any recursion operator $R$ for $X$ is such that $R X$ is parallel to $X$. This raises some questions about the ability to generate new symmetries for degenerate systems by recursion operators. We extend here this result to systems with quasi-periodic motions:

Proposition A.2. Consider a vector field $X$ which is Hamiltonian and noncommutatively integrable relative to a symplectic structure $\sigma_{0}$ and which has $n$ frequencies; let $\pi$ be the fibration by its invariant tori. Assume that $X$ is Hamiltonian relative to another symplectic structure $\sigma_{1}$ and let $R=\sigma_{0}^{\sharp} \sigma_{1}^{b}$. If (i) $\sigma_{0}$ and $\sigma_{1}$ are $\left(\pi, \pi^{\perp}\right)$-compalible, or (ii) $X$ is anisochronous, then $R^{-1} X$ is tangent to the fibers of $\pi$.

Proof. In $\sigma_{0}$-generalized action-angle coordinates, we have $X=\omega_{i}(a) \partial / \partial \alpha_{i}=\sigma_{0}^{\sharp}(-\mathrm{d} H)$. Preliminarily, let us note that, under any of the two hypotheses (i) and (ii), $\sigma_{1}$ has the form (2.10). This follows from statement (i) of Proposition 1 in case (i) and from statement (i.2) of Proposition 2 in case (ii). Hence, if we let $Y=R^{-1} X$, we have

$$
\sigma_{1}^{\mathrm{b}}(Y)=E_{j i} Y^{a_{j}} \mathrm{~d} \alpha_{i}-\left(E_{j i} Y^{\alpha_{i}}-F_{r, 2 d-2 n+j} Y^{b_{r}}\right) \mathrm{d} a_{j}+F_{r s} Y^{b_{r}} \mathrm{~d} b_{s}
$$


(here $s$ takes the values $1, \ldots, 2 d-2 n$, while all other indices obey our previous conventions). But $\sigma_{1}^{b}(Y)=-\mathrm{d} H=-\omega_{i}(a) \mathrm{d} a_{i}$, and so

$$
E_{j i} Y^{a_{j}}=0, \quad E_{j i} Y^{\alpha_{i}}-F_{r, 2 d-2 n+j} Y^{b_{r}}=-\omega_{j}, \quad F_{r s} Y^{b_{r}}=0
$$

for all $j=1 \ldots, n, r=1, \ldots, 2 d-n$, and $s=1, \ldots, 2 d-2 n$. Since $E$ is invertible, the first equation implies $Y^{a}=0$. Therefore, the last equation becomes

$$
\left(\begin{array}{cc}
F_{p p} & F_{p q} \\
-F_{p q}^{\mathrm{T}} & F_{q q}
\end{array}\right)\left(\begin{array}{c}
Y^{p} \\
Y^{q}
\end{array}\right)=0,
$$

where the matrix is invertible by the hypotheses $(2.8)$ on the ranks of $E$ and $(F, E)$. We conclude $Y^{b}=0$ and thus $Y$ is tangent to the fibers of $\pi$.

\section{Appendix B}

In this appendix we make some comments on Definition 2 . We begin by showing that the three conditions entering (D2.1) are independent of each other. First, the following example shows that the requirement of bi-affinity is independent of the other two:

$$
\begin{array}{ll}
M=\mathbb{R} \times S^{1} \ni(a, \alpha), & \sigma_{0}=\mathrm{d} a \wedge \mathrm{d} \alpha \\
\pi(a, \alpha)=a, & \sigma_{1}=\left(1+\frac{1}{2} \sin \alpha\right) \mathrm{d} a \wedge \mathrm{d} \alpha .
\end{array}
$$

The fibers of $\pi: M \rightarrow \mathbb{R}$ are bi-Lagrangian (hence bi-isotropic and bi-symplectically complete), but

$$
\nabla_{\partial / \partial \alpha}^{\left(\sigma_{0}\right)} \frac{\partial}{\partial \alpha}=0, \quad \nabla_{\partial / \partial \alpha}^{\left(\sigma_{1}\right)} \frac{\partial}{\partial \alpha}=\frac{\cos \alpha}{2+\sin \alpha} \frac{\partial}{\partial \alpha}
$$

(Incidentally, $(a, \alpha)$ and $\left(a, \alpha-\frac{1}{2} \cos \alpha\right)$ are $\sigma_{0}$ - and $\sigma_{1}$-action-angle coordinates of $\pi$, respectively.)

Second, the following example shows that the $\sigma_{0}$ - and $\sigma_{1}$-polars of $\pi$, when they exist, need not coincide

$$
\begin{aligned}
& M=\mathbb{R} \times \mathbb{R} \times \mathbb{R} \times S^{1} \ni(p, q, a, \alpha), \quad \sigma_{0}=\mathrm{d} a \wedge \mathrm{d} \alpha+\mathrm{d} p \wedge \mathrm{d} q, \\
& \pi(p, q, a, \alpha)=a, \quad \sigma_{1}=\mathrm{d}\left(a^{2}+p^{2}\right) \wedge \mathrm{d} \alpha+\mathrm{d} p \wedge \mathrm{d} q .
\end{aligned}
$$

The fibration $\pi: M \rightarrow \mathbb{R}$ is clearly bi-isotropic; it is also bi-affine, since $\partial / \partial \alpha=X_{\left(a^{2}+p^{2}\right)}^{\sigma_{1}}$ and so

$$
\nabla_{\partial / \partial \alpha}^{\left(\sigma_{1}\right)} \frac{\partial}{\partial \alpha}=\sigma_{1}^{\sharp}\left(\frac{\partial}{\partial \alpha}\left(a^{2}+p^{2}\right)\right)=0
$$

However, the leaves of the $\sigma_{0}$ - and $\sigma_{1}$-polar foliations of $\pi$ are given by the equations $a=$ const. and $a^{2}+p^{2}=$ const., respectively.

Third, it is well known that not every isotropic fibration has a polar foliation [16, Section 4.6]. 
The next Proposition contains a number of equivalent restatements of the condition of bi-affinity, which should help clarify its meaning:

Proposition B.1. Let $\pi: M \rightarrow B$ be a fibration with compact and connected fibers which is isotropic and symplectically complete with respect to two symplectic structures $\sigma_{0}$ and $\sigma_{1}$ on $M$. Then, the following conditions are equivalent:

(i) The restrictions of $\nabla^{\left(\sigma_{0}\right)}$ and of $\nabla^{\left(\sigma_{1}\right)}$ to the fibers of $\pi$ coincide.

(ii) In a neighborhood of every fiber of $\pi$ there exist $n$ everywhere linearly independent $\sigma_{0}$ - and $\sigma_{1}$-Hamiltonian vector fields which are tangent to the fibers of $\pi$.

(iii) In a neighborhood of every fiber of $\pi$ there exist $\sigma_{0}$ - and $\sigma_{1}$-generalized action-angle coordinates of $\pi,(p, q, a, \alpha)$ and $(x, y, e, \eta)$, respectively, such that $X_{a_{i}}^{\left(\sigma_{0}\right)}=X_{e_{i}}^{\left(\sigma_{1}\right)}$.

(iv) In a neighborhood of every fiber of $\pi$ there exists a vector field which is both $\sigma_{0}$ - and $\sigma_{1}$-Hamiltonian, is tangent to the fibers of $\pi$, and has $n$ frequencies.

Proof. We denote by $(b, \alpha)=(p, q, a, \alpha)$ a system of $\sigma_{0}$-generalized action-angle coordinates of $\pi$ and by $X_{H}^{\sigma_{i}}$ the $\sigma_{i}$-Hamiltonian vector field of a function $H$.

(i) $\Rightarrow$ (iii): Proceeding as in the proof of statement (i) of Proposition 1, one finds that $\sigma_{1}$ has expression (2.6), so that $\partial / \partial \alpha_{i}=X_{a_{i}}^{\sigma_{0}}=X_{e_{i}}^{\sigma_{1}}$.

(ii) $\Rightarrow$ (i): Consider any symplectic manifold $(M, \sigma)$ and an isotropic symplectically complete fibration of $M$ with compact connected fibers. Let $\nabla^{(\sigma)}$ denote the associated partial connection given in (2.1). Then, if $X$ and $Y$ are any two Hamiltonian vector fields tangent to the fibers of this fibration, one has $\nabla_{X}^{(\sigma)} Y=0$ (this is seen by referring to (2.3) and using the fact that the Hamiltonian of $Y$ depends only on the actions $a$ ). Hence, if $Y_{1}, \ldots, Y_{n}$ are as in hypothesis (ii), then $\nabla_{Y_{i}}^{\left(\sigma_{0}\right)} Y_{j}=\nabla_{Y_{i}}^{\left(\sigma_{1}\right)} Y_{j}=0$ for all $i, j$. This shows that $\nabla^{\left(\sigma_{0}\right)}$ and $\nabla^{\left(\sigma_{1}\right)}$ agree on a basis for the sections of $\pi$, and so their restrictions to the fihers of $\pi$ are equal.

(i) $\Rightarrow$ (iv): For any nonresonant $\omega \in \mathbb{R}^{n}$, the vector field $\omega_{i} \partial / \partial \alpha_{i}$ has all the properties stated in (iv). (It is $\sigma_{1}$-Hamiltonian because so are the vector fields $\partial / \partial \alpha_{i}$, as proven in (i) $\Rightarrow$ (iii) above.)

Finally, note that (iii) $\Rightarrow$ (ii) is obvious while (iv) $\Rightarrow$ (i) follows from statement (i) of Proposition 2.

\section{References}

[1] J.H. Wilkinson, Note on matrices with a very ill-conditioned eigenproblem, Numer. Math. 19 (1972) 176-178.

[2] F. Magri, A simple model of the integrable Hamiltonian equations, J. Math. Phys. 19 (1978) 1156-1162.

[3] R. Brouzet, About the existence of recursion operators for completely integrable systems near a Liouville torus, J. Math. Phys. 34 (1993) 1309-1313.

[4] R.L. Fernandes, Completely integrable bi-Hamiltonian systems, J. Dynam. Differential Equations 6 (1994) 53-69.

[5] R. Brouzet, P. Molino, F.J. Turiel, Géométrie des systèmes bihamiltoniens, Indag. Math. 4 (1993) 269296.

[6] O.I. Bogoyavlenskij, Theory of tensor invariants of integrable Hamiltonian systems, I. Incompatible Poisson structures, Comm. Math. Phys. 180 (1996) 529-586. 
[7] F. Fassò, Quasi-periodicity of motions and complete integrability of Hamiltonian systems, Ergodic Theory and Dynamical Systems, to appear.

[8] V.I. Arnold, Méthodes Mathématiques de la Mécanique Classique, MIR, Moscow, 1976.

[9] F. Fassò, The Euler-Poinsot top: a non-commutatively integrable system without global action-angle coordinates, J. Appl. Math. Phys. (ZAMP) 47 (1996) 953-976.

[10] J.F. Cariñena, L.A. Ibort, On the Lax equations arising from Lagrangian foliations, Lett. Math. Phys. 8 (1984) 21-26.

[11] Y. Kosmann-Schwarzbach, Lagrangian foliations and Lax equations, Lett. Math. Phys. 9 (1985) 163167.

[12] M.V. Karasev, V.P. Maslov, Nonlinear Poisson Brackets. Geometry and Quantization, Translations of the AMS, vol. 119, AMS, Providence, RI, 1993.

[13] A. Weinstein, The local structure of Poisson manifolds, J. Differential Geom. 18 (1983) 523-557.

[14] P. Libermann, C.-M. Marle, Symplectic Geometry and Analytical Mechanics, Reidel, Dordrecht, 1987.

[15] P. Dazord, T. Delzant, Le problème général des variables actions-angles, J. Differential Geom. 26 (1987) 223-251.

[16] N.M.J. Woodhouse, Geometric Quantization, 2nd ed., Clarendon Press, Oxford, 1994.

[17] N.N. Nekhoroshev, Action-angle variables and their generalizations, Trudy Moskov. Mat. Obsc. 26 (1972) 181-198; Trans. Moskow Math. Soc. 26 (1972) 180-198.

[18] O.I. Bogoyavlenskij, A concept of integrability of dynamical systems, C. R. Math. Rep. Acad. Sci. Canada 18 (1996) 163-168.

[19] O.I. Bogoyavlenskij, Theory of tensor invariants of integrable Hamiltonian systems, II, Theorem on symmetries and its applications, Comm. Math. Phys. 184 (1996) 301-365.

[20] G. Marmo, G. Vilasi, When do recursion operators generate new conservation laws?, Phys. Lett. B 277 (1992) 137-140.

[21] S.L. Ziglin, Nonintegrability of a problem on the motion of four point vortices, Sov. Math. Dokl. 21 (1980) 296-299.

[22] J. Koiller, S. Pinto de Carvalho, Nonintegrability of the 4-vortex system: analytical proof, Comm. Math. Phys. 120 (1989) 643-652.

[23] M.S.A.C. Castilla, V. Moauro, P. Negrini, W.M. Oliva, The four positive vortices problem: regions of chaotic behavior and the nonintegrability, Ann. Inst. Henri Poincaré - Physique Thèorique 59 (1993) 99-115.

[24] F. Fassò, T. Ratiu, paper in preparation.

[25] Y. Kosmann-Schwarzbach, Géométrie des systèmes bihamiltoniens. Sem. Math. Sup. 102 (1986) 185216.

[26] G. Gallavotti, private communication. 